\title{
Geometry Control of Closed Contour Forming in Uniform Micro Metal Droplet Deposition Manufacturing
}

Daicong Zhang ${ }^{1}$, Lehua Qi ${ }^{12^{*}}$, Jun Luo ${ }^{1,2^{*}}$, Hao $\mathrm{Yi}^{1}$, Xianghui Hou ${ }^{3}$, Hejun $\mathrm{Li}^{4}$

1. School of Mechanical Engineering, Northwestern Polytechnical University, Xi'an 710072, China.

2. Education Ministry Key Laboratory of Modern Design and Integrated Manufacturing Technology, Northwestern Polytechnical University, Xi'an 710072,China

3. Faculty of Engineering, University of Nottingham, Nottingham NG7 2RD, United Kingdom.

4. School of Material, Northwestern Polytechnical University, Xi'an 710072, China

\section{Abstract:}

Micro metal droplet deposition manufacturing shows great potential applications in many industrial areas such as micro circuits printing, thin-wall metal parts, porous metal parts, and heterogeneous material parts. However, excessive overlapping of metal droplets in corners deteriorates the quality of printed parts. To solve this problem, the droplet center-to-center distance must always keep uniform and be in an ideal range. First, reasons of excessive overlapping in corners are analyzed and a mathematical model is proposed. Then droplet center-to-center distance is optimized and compensated according to corner angle of contour lines and number total of droplets so that the distance between adjacent droplets is proper. The coordinate of rearranged droplets is obtained by calculation. To verify this method, uniform solder droplets were 
ejected and deposited on a flat substrate. A series of deposition experiments were carried out. Some triangle contours were formed. The results show that quality of the formed parts had been significantly improved by using the proposed method.

Keywords: excessive overlapping; corners; contour forming; uniform micro metal droplet deposition manufacturing

\section{Introduction}

Micro metal droplet deposition manufacturing is an additive manufacturing technology in which 3D metal parts are fabricated by printing molten metal droplets. In the process, molten metal droplets are generated to form a metal part directly without expensive energy source or special powder. Molten micro droplets deposition process was first put forward by Gao et al. (1994). Later, uniform metal droplets printing apparatuses were developed. Orme et al. (2000) developed molten aluminum alloy droplet generator for application to net-form manufacturing of structural components. Orme et al. (2000) studied enhanced aluminum properties by means of precise droplet deposition. Liu et al. (2001) introduced the principle of the solder droplet printing technology and potential application of the technology in microelectronics industry. Molten metal droplets ejected by a pneumatic droplet-on-demand generator were investigated 
by Cheng et al. (2005). Some samples such as straight lines, circles, triangles, grids were formed by the deposition of molten metal droplets by Fang et al. (2008). Then Fang et al. (2009) discussed heat transfer during deposition of molten aluminum alloy droplets to build vertical columns. Although several rapid prototyping systems based on metal droplet had been developed in recent years to produce various parts, some geometry control problems still exist, such as overlapping parameter of adjacent droplets, uniform height of contour, and contour closure. These problems will affect the quality and accuracy of the formed parts.

Furthermore, the above problems should exist in droplet deposition of the other materials, as long as they are made of solidifying matter (e.g. polymers and metals) or even yield stress materials (e.g. hydrogels). The problem is relevant for a wide range of scales : (1) $1 \sim 10 \mu \mathrm{m}$ droplets, as produced with Laser-induced Forward Transfer (LIFT), which is applied in bio-printing (Mézel et al., 2010), Deoxyribonucleic acid (Colina et al., 2005; Serra et al., 2004) and metal Nano-paste deposition (Visser, 2015; Zenou et al., 2015). (2) 10 100 $\mu \mathrm{m}$ metal and hydrogel droplets (Li et al., 2011; Um et al., 2008), as achieved with inkjet printing. (3) $100 \sim 1000 \mu \mathrm{m}$ metal droplets, as achieved with recently developed metal printers, which is concerned by this paper. The addressed problem will benefit each solidifying droplet-based technique for 3D printing, so the urgency and broad scope is not limited to 
metal droplets. Subsequently, these problems about geometry control of closed contour forming will be addressed with a model material (Sn63\% Pb37\% metal alloy) in this paper which can be reproducibly ejected as a droplet.

In the droplet deposition process, a single droplet is used as a manufacturing unit. The size of the metal droplet can't be changed. Therefore, re-arranging the position of every micro droplet is an effective method to eliminate the excessive overlapping in corners which was put forward by Horii et al. (2009), but the rearranged method has a great influence on the forming accuracy, because actual scanning path could stray away from the ideal path. Profile error and extruding aperture for the RP (rapid prototyping) parts were studied using the fused deposition modeling process by Chang et al. (2011). Algorithm for detecting and solving the problem of under-filled pointed ends based on 3D printing plastic droplet generation was also developed by Jelena Prša et al. (2014). However, there are still some problems about overlapping in corners, uniformity of contour lines, and contour closure and so on. The above several issues about effective geometry control of closed contour forming still need to be solved. First, the reasons of excessive overlapping in corners of contour segments needs to be analyzed. Second, the contour closure strategy needs to be studied.

In uniform micro metal droplet deposition manufacturing process, if the droplet center-to-center distance is controlled in a proper range, it is possible to 
eliminate the excessive overlapping in corners. First, reasons of excessive overlapping in corners is analyzed and a mathematical model is proposed. Then the droplet center-to-center distance is compensated according to the corner angle of contour line and the droplet total number in contour line, and the coordinate of rearranged droplets is obtained.

\section{Experimental System and Method}

Uniform micro metal droplet deposition manufacturing system is shown in Fig. 1, which is explored by our team formerly (Luo et al., 2008). It mainly consists of a three-dimensional platform, a metal melting crucible, a protective gas system, a pulse generator, a temperature measurement system, and a substrate heating system. The Numerical Control (NC) code generated by the host computer is downloaded into the Programmable Multi Axis Controller (PMAC), which controls the three-dimensional platform to go to the corresponding positions, then triggers the piezoelectric ceramic. The stress wave is produced by micro vibration of the bar in the metal liquid. On the peak of the stress wave, a small amount of liquid is squeezed out of the nozzle. On the trough of the stress wave, the metal liquid near the nozzle is retracted and separated from the metal liquid out of the nozzle. Micro metal droplets are ejected onto the substrate out of the micro-sized nozzle. On the moment of colliding with the substrate, a hemispherical droplet is formed, the diameter of which is slightly larger than the diameter of the original one. Then the three-dimensional platform 
is located to the next point position and the next metal droplet is ejected. Subsequently, the part is piled up layer by layer.

Uniform micro metal droplets were ejected by the above experiment system. The droplet material was $\mathrm{Sn} 63 \% \mathrm{~Pb} 37 \%$. The initial temperature of droplet was 573K. Substrate temperature was 293 298K. Back pressure was 3.0 5.0 kPa. Pulse width time was $1 \mathrm{~ms}$. Oxygen content of environment was 50 60 PPM (parts per million). Nozzle diameter was $300 \mu \mathrm{m}$.

The parameters involved in the calculation are shown in Table 1.

\section{Theoretical Analysis and Results}

\subsection{Analysis of excessive overlapping in corners}

The droplet center-to-center distance between two adjacent droplets is also called scanning step distance. If the scanning step distance is too small, excessive overlapping would occur. If the step distance is too far, partial overlapping takes place. The scanning step distance will be the best, when the merging height of the two adjacent droplets just is equal to the highest point of the hemispherical droplets. 
The droplet impacted and solidified on the substrate is defined as a spherical cap. The theoretical value of the ideal step distance is calculated by the solidification angle and the diameter of the droplet just ejected from the nozzle (Qi et al., 2012).

In the ideal state, the distance between every two adjacent droplets is in the ideal range, but in corners, the smaller the corner angle is, the smaller the actual distance between two adjacent droplets is than the ideal distance. As shown in Fig. 2, the actual step distance $\mathrm{c}^{\prime}$ is smaller than the sum of $a$ and $b$ due to $a, b$ and $c^{\prime}$ forming a triangle. The actual step distance $c^{\prime}$ must be smaller than the ideal step distance $c$.

Without considering the change of the speed in Numerical Control (NC) system, the actual step distance between two adjacent droplets in corners could be represented by equation (1).

$$
c^{\prime}=\sqrt{(c-b)^{2}+b^{2}-2(c-b) \times b \times \cos \frac{\pi \theta}{180}}
$$

Where, $b$ is the distance from the end droplet to the inflection point, $c$ is the ideal step distance, $c^{\prime}$ is the actual step distance, and $\theta$ is the corner angle.

A series of corner angles have been used: $0^{\circ}, 30^{\circ}, 45^{\circ}, 60^{\circ}, 90^{\circ}, 120^{\circ}, 150^{\circ}$ and $180^{\circ}$. The related curves were drawn according to the distance from the end 
droplet to the inflection point $b$ as abscissa and the actual distance $c^{\prime}$ between two adjacent droplets as ordinate, as shown in Fig. 2, and the ideal step distance c is supposed to $200 \mu \mathrm{m}$.

In the process of forming corners, because of the excessive overlapping of two adjacent droplets, the actual distance between the centers of two adjacent droplets in corners is smaller than the ideal step distance. When the corner angle is smaller or $b$ is closer to half of the ideal step distance, the excessive overlapping is much more serious in corners.

To solve the problem of excessive overlapping, two conditions need to be met: (i) The location of droplet deposition is still on the scanning line; (ii) The actual distance between two adjacent droplets in corners is in the ideal range.

When the corner angle is in the ranges of $0^{\circ} \sim 45^{\circ}$, or $45^{\circ} \sim 90^{\circ}$ and $b<c \cdot \cot \theta$, the starting distance $a$ and the ideal step distance $c$ could be represented by equation (2).

$$
a=\frac{c}{\sin \theta}
$$

When the corner angle is $90^{\circ} \sim 180^{\circ}$, or $45^{\circ} \sim 90^{\circ}$ and $b>c^{\circ} \cot \theta$, It could be represented by equation (3), as shown in Fig. 3. 


$$
a=b \cos \theta+\sqrt{(b \cos \theta)^{2}-\left(b^{2}-c^{2}\right)}
$$

3.2 Analysis of excessive overlapping in the contour closure

The majority of 3D printed objects have a lot of closed contours, which can only be filled with an integer number of droplets in uniform micro metal droplet deposition manufacturing. However, the end droplet and the first droplet is possibly partial or excessive overlapping. Therefore, in order to prevent this type of defect, a method is proposed to slightly adjust the droplet droplet-to-droplet distance.

As shown in Fig. 4, excessive overlapping in corners could be solved by adjusting scanning step distance except in the contour closure. Scanning step distance is a constant in contour lines. If the distance between the end droplet and the first droplet of a contour closure is not in ideal range, excessive overlapping would occur in the contour closure.

To solve the problem, a compensation method is used to adjust the step distance. And compensation values are equally distributed to the distance between every two droplets. So forming quality will be slightly affected.

Firstly, the number of droplets on the current line $L_{i}$ is obtained by the optimized scanning step distance. Then the distance between the end droplet and 
the first droplet at the contour closure is determined. At last, the excessive overlapping would be eliminated by the step distance compensation.

The number $m_{i}$ of droplets needed for the current line $i$ :

$$
m_{i}=\operatorname{int}\left(\frac{L_{i}-a_{i}}{c}\right)
$$

Where, mi is the droplet number on the current line $i, L_{i}$ is the length of current line, $a_{i}$ is the distance from the starting point of the current line $i$ to the first droplet center.

The sum of $a_{i}$ and all the step distance is equal to the length from the starting point to the current droplet $j$ :

$$
a_{i}+j \cdot c=\sqrt{\left(x_{j}-x_{-} s\right)^{2}+\left(y_{j}-y_{-} s\right)^{2}}
$$

Where, $\left(x \_s, y_{-} s\right)$ is the coordinate of the starting point of line $i,\left(x_{j}, y_{j}\right)$ is the coordinate of the current droplet $j$ in line $i$.

$$
\frac{y_{j}-y_{-} s}{x_{j}-x_{-} s}=\frac{y_{-} e-y_{-} s}{x_{-} e-x_{-} s}=k
$$

Where, $\left(x \_s, y \_s\right)$ is the coordinate of the end point of line $i, k$ is the slope of line $i$.

Combining equation (5) and (6), the coordinate equation of droplet $j$ in line $i$ : 


$$
\left\{\begin{array}{l}
x_{j}=x_{-} s+\frac{a_{i}+j \cdot c}{\sqrt{1+k^{2}}} \\
y_{j}=k x_{j}-k x_{-} s+y_{-} s
\end{array} \quad\left(j<m_{i}\right)\right.
$$

The distance $b_{i}$ between the end point of current line $i$ and the end droplet:

$$
b_{i}=L_{i}-m_{i} \cdot c
$$

$a_{i+1}$ of line $i+1$ is calculated by $b_{i}$ and $\theta$ :

$$
a_{i+1}= \begin{cases}\frac{c}{\sin \theta} & \theta=0^{\circ} \sim 45^{\circ} \text { or } 45^{\circ} \sim 90^{\circ} \text { and } b_{i}<\cot \theta \\ b_{i} \cdot \cos \theta+\sqrt{\left(b_{i} \cdot \cos \theta\right)^{2}-\left(b_{i}^{2}-c^{2}\right)} & \theta=90^{\circ} \sim 180^{\circ} \text { or } 45^{\circ} \sim 90^{\circ} \text { and } b_{i}>\cot \theta\end{cases}
$$

Combining equation (4) (8), all the droplet coordinate and $b_{i+1}$ can be calculated in next scanning line $i+1$.

The compensated step distance $c_{c m p}$ :

$$
c_{c m p}=c-\frac{a_{n}}{N}
$$

Where, $a_{n}$ is the distance from the starting droplet of the first line to the end point of the last line, $N$ is the number of all the droplets on contour.

When the compensated step distance is close to the ideal step distance, the contour is fully closed. When the contour is longer, the error e between the 
compensated step distance and the ideal step distance becomes smaller, as shown in Fig. 5.

The error e between the compensated step distance and the ideal step distance:

$$
e=c-c_{c m p}=\frac{a_{n}}{N}
$$

where

$$
0<a_{n}<c \Rightarrow 0<e<\frac{c}{N}
$$

As the droplet diameter is micron-sized, and the length of the contour line is centimeter-sized generally, the error could be neglected.

\section{Experimental Results}

In the process of forming closed contour parts, the geometry shape are mainly affected by the nozzle diameter, material, droplet spreading and substrate temperature, etc. In different droplet generation techniques, such as LIFT, the theory still holds.

The sample is formed by continuous lines. Corners are composed of adjacent lines. In order to investigate the effect of process parameters on corners, a series 
of experiments were carried out. Firstly, deposition morphology of single droplet ejected from a $300 \mu \mathrm{m}$ diameter nozzles was analyzed in order to obtain the proper scanning step distance. Then, a closed triangle contour before and after optimization with three layers was formed.

4.1 Determination of optimal step distance $c$

Solidification angle $\theta_{\text {sld }}$ is the angle which is formed by tangent line at the point contacting with substrate. $\alpha$ is complementary angle of $\theta_{\text {sld }}$.

$$
\theta_{\text {sld }}=\pi-a
$$

Where, the angle $\alpha$ is obtained by height $h$ and spreading radius $R$, as shown in Fig. 6(a).

$$
\alpha=\arccos \frac{h-R}{R}
$$

Where, $h$ is the height of spreading droplet; $R$ is the radius of spreading droplet.

At the case of the ideal step distance, the shape of merger part is a rectangle at the interface. Width of the rectangle is the ideal step distance $c$, height of the rectangle is height $h$ of the spreading droplet, area of the rectangle $S_{A B C D}$ is area 
of a single droplet $S_{\text {droplet, }}$ as shown in Fig. 6. The ideal step distance $c$ is obtained by equation (15).

$$
c=\frac{S_{A B C D}}{h}=\frac{S_{\text {droplet }}}{h}=\frac{(h-R) R \sin \frac{\theta_{\text {sld }}}{2}+\frac{(2 \pi-2 \alpha) R^{2}}{2}}{h}
$$

The droplet spreading radius $\mathrm{R}$ was $183 \pm 10 \mu \mathrm{m}$. The droplet spreading height $h$ was $345 \pm 5 \mu \mathrm{m}$. The droplet solidification angle $\theta_{\text {sld }}$ was $108^{\circ}$. Step distance $c$ in the theory was $252.5 \mu \mathrm{m}$.

\subsection{Closed contour forming}

A triangle contour with 3 layers was formed. The triangle angle is respectively $30^{\circ}, 60^{\circ}, 90^{\circ}$. The side length of the triangle is respectively $3 \mathrm{~mm}$, $6 \mathrm{~mm}, 5.196 \mathrm{~mm}$. The unoptimized sample was shown in Fig. 7(a), the sample optimized by the corner calculation was shown in Fig. 7(b) and the sample optimized by the compensation calculation for closed contour was shown in Fig. 7(c).

The parameters involved in the calculation are shown in Table 2. In no optimization, $a$ and $b$ is equal to $c$; In the corners optimization, $m_{i}, a_{i}$ and $b_{i}$ were calculated by equation (4) and (9); In the contour optimization, $c_{c m p}, m_{i}, a_{i}$ and $b_{i}$ were calculated by equation (4), (9) and (11). The overlapping error of 
the final droplet was $7.5 \mathrm{pm}$. The distance between the starting droplet and the end droplet was fallen from $0.183 \mathrm{~mm}$ to $0.027 \mathrm{~mm}$.

When the corner angle of the triangle was $30^{\circ}$, the measurement was shown that maximum height of the unoptimized sample was about $795 \mathrm{pm}$, and maximum height of the optimized sample was about $597 \mathrm{pm}$. Difference of the maximum height in corners $\left(30^{\circ}\right)$ is about $98 \mathrm{pm}$. Height difference of a single layer is about $32.6 \mathrm{pm}$. When the corner angle of the triangle was $60^{\circ}$, the measurement was shown that maximum height of the unoptimized sample was about $652 \mathrm{pm}$, and maximum height of the optimized sample was about $558 \mathrm{pm}$. Difference of the maximum height in corners $\left(60^{\circ}\right)$ is about $94 \mathrm{pm}$. Height difference of a single layer is about $31.3 \mathrm{pm}$. Quality of the final formed parts have been improved a lot with the help of the algorithm, especially in corners. In no contour optimization, the distance between the starting droplet and the end droplet was not equal to the ideal scanning distance, as shown in Fig. 7(b). Height of excessive overlapping was $796 \mathrm{pm}$, which is far higher than the mean overlapping height. After the contour optimization, excessive overlapping in contour closure had been eliminated, as shown in Fig. 7(c). The optimized triangle contour can be fully closed and excessive overlapping does not occur in corners or the end point. 


\section{Conclusions}

This paper analyzes excessive overlapping of the corners at the contoursegments in uniform micro metal droplet deposition manufacturing. The efficiency of the control strategy has been verified by experiments.

1) The unoptimized actual step distance is smaller than the ideal step distance in corners. When the corner angle is smaller or distance from the droplet center to the inflection point is closer to half of the ideal step distance, the excessive overlapping is more serious in corners.

2) The mathematical model used to solve the problem of excessive overlapping in corners is effective. Two conditions could to be met: (i)The location of droplet deposition is still on the scanning line; (ii)The actual distance between two adjacent droplets in corners is in the optimal range.

3) The longer the contour is, the smaller the overlapping error of the final droplet due to non-integer of contour droplet number is. As the droplet diameter is micron-sized, and the length of the contour line is centimetersized generally, the error could be neglected.

4) The results of experiments demonstrates that a triangle contour can be fully closed by the geometry control strategy. Quality of the final formed 
parts have been improved a lot with the help of the compensation method, especially in corners.

5) The compensation method could be applicable to droplet disposition of the other materials, as long as they are made of solidifying matter (e.g. polymers and metals) or even yield stress materials (e.g. hydrogels).

\section{Acknowledgments}

This work was supported by National Natural Science Foundation of China

(No. 51675436), Natural Science Basic Research Plan in Shanxi Province of China (No. 2015JQ5123), Programme of Introducing Talents of Discipline to Universities (Grant No. B08040).

\section{References:}

Chang, D.-Y., Huang, B.-H., 2011. Studies on profile error and extruding aperture for the RP parts using the fused deposition modeling process. The International Journal of Advanced Manufacturing Technology 53, 1027-1037.

Cheng, S.X., Li, T., Chandra, S., 2005. Producing molten metal droplets with a pneumatic droplet-on-demand generator. Journal of Materials Processing Technology 159, 295-302. Colina, M., Serra, P., Fernández-Pradas, J.M., Sevilla, L., Morenza, J.L., 2005. DNA deposition through laser induced forward transfer. Biosensors and Bioelectronics 20, 1638-1642.

Fang, M., Chandra, S., Park, C.B., 2008. Building three-dimensional objects by deposition of molten metal droplets. Rapid Prototyping Journal 14, 44-52.

Fang, M., Chandra, S., Park, C.B., 2009. Heat transfer during deposition of molten aluminum alloy droplets to build vertical columns. Journal of Heat Transfer 131, 1-7.

Gao, F., Sonin, A.A., 1994. Precise deposition of molten microdrops: the physics of digital microfabrication. Proceedings of the Royal Society of London. Series A: Mathematical and Physical Sciences 444, 533-554. 
Horii, T., Kirihara, S., Miyamoto, Y., 2009. Freeform fabrication of superalloy objects by 3D micro welding. Materials \& Design 30, 1093-1097.

Jelena Prša, F.I., Tim C. Lueth, 2014. Algorithm for Detecting and Solving the Problem of Under-Filled Pointed Ends Based on 3D Printing Plastic Droplet Generation. ASME 2014 International Mechanical Engineering Congress and Exposition 2A.

Li, H., Leulmi, R.F., Juncker, D., 2011. Hydrogel droplet microarrays with trapped antibody-functionalized beads for multiplexed protein analysis. Lab on A Chip 11, 528-534. Liu, Q., Melissa Orme, Orme, M., 2001. High precision solder droplet printing technology and the state-of-the-art. Journal of Materials Processing Technology 115, 271-283.

Luo, J., Qi, L.H., Jiang, X.S., Zhou, J.M., Huang, H., 2008. Research on lateral instability of the uniform-charged droplet stream during droplet-based freeform fabrication. International Journal of Machine Tools \& Manufacture 48, 289-294.

Mézel, C., Souquet, A., Hallo, L., Guillemot, F., 2010. Bioprinting by laser-induced forward transfer for tissue engineering applications: jet formation modeling. Biofabrication 2, 65-117.

Orme, M., Liu, Q., Fischer, J., 2000. Mono-disperse aluminum droplet generation and deposition for net-form manufacturing of structural components, Eighth International Conference on Liquid Atomization and Spray Systems, pp. 200-207.

Orme, M., Smith, R.F., 2000. Enhanced aluminum properties by means of precise droplet deposition. Transactions Ransactions-american Society of Mechanical Engineers Journal of Manufacturing Science and Engineering 122, 484-493.

Qi, L.-h., Chao, Y.-p., Luo, J., Zhou, J.-m., Hou, X.-h., Li, H.-j., 2012. A novel selection method of scanning step for fabricating metal components based on microdroplet deposition manufacture. International Journal of Machine Tools and Manufacture 56, 50-58.

Serra, P., Colina, M., Fernándezpradas, J.M., Sevilla, L., Morenza, J.L., 2004. Preparation of functional DNA microarrays through laser-induced forward transfer. Applied Physics Letters 85, 1639-1641.

Um, E., Lee, D.S., Pyo, H.B., Park, J.K., 2008. Continuous generation of hydrogel beads and encapsulation of biological materials using a microfluidic droplet-merging channel. Microfluidics \& Nanofluidics 5, 541-549.

Visser, C.W., 2015. Toward 3D Printing of Pure Metals by Laser-Induced Forward Transfer. Advanced Materials 27, 4087-4092.

Zenou, M., Sa'Ar, A., Kotler, Z., 2015. Laser Transfer of Metals and Metal Alloys for Digital Microfabrication of 3D Objects. Small 11, 1-9. 


\section{Figures:}

Fig. 1 Schematic diagram of uniform micro metal droplet deposition manufacturing experimental system.

Fig. 2 The actual step distance under different angles $\theta$ and different distances from droplet center to inflection point $b$.

Fig. 3 Geometry control at different angles.

Fig. 4 Rearrange for droplets position of contour closure

(a)Excessive overlapping in the contour closure; (b) Contour formed by compensated optimal step distance.

Fig. 5 Error of step distance after optimized.

Fig. 6 The samples with different corner angle.

Fig. 7 Optimization of a triangle sample.

(a) No optimization; (b) Corner optimization; (c) Contour optimization 


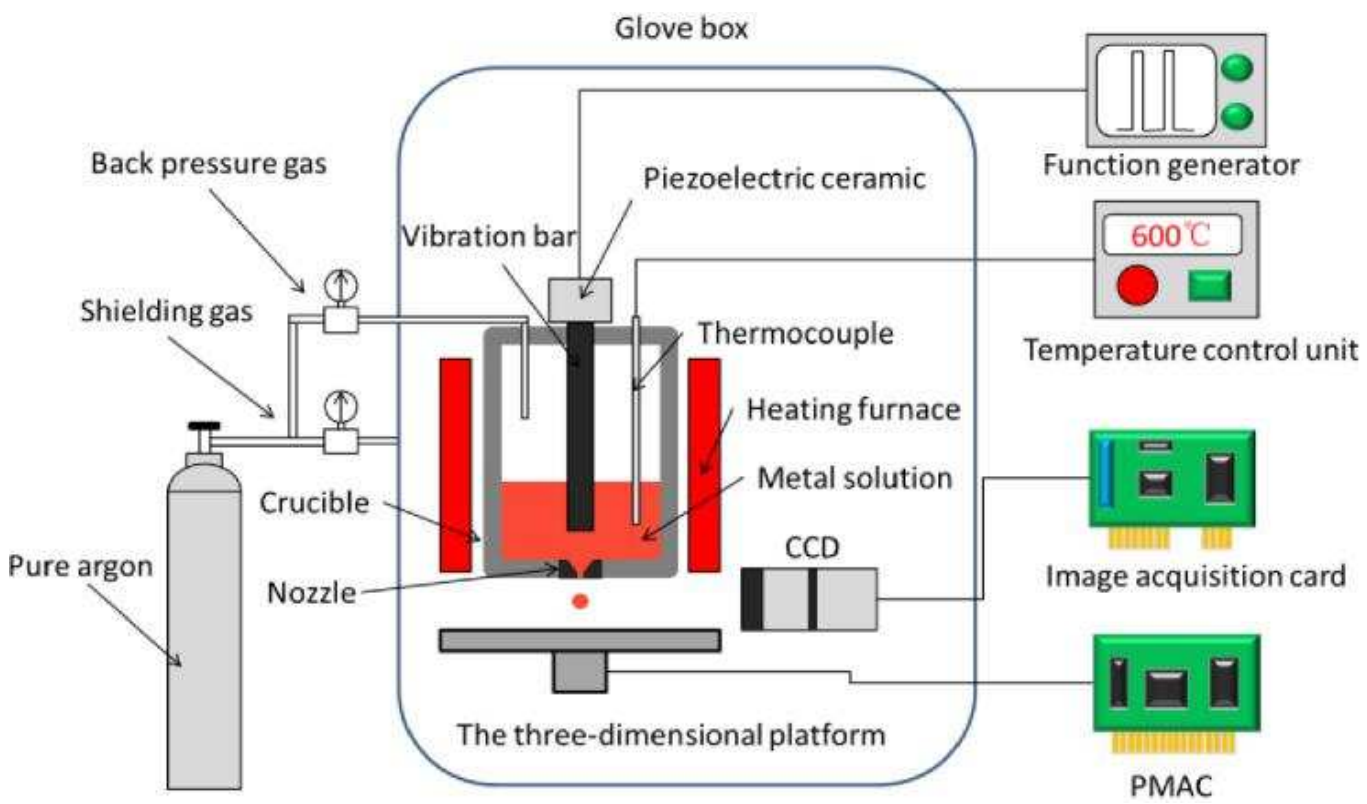

Fig. 1 Schematic diagram of uniform micro metal droplet deposition manufacturing experimental system. 


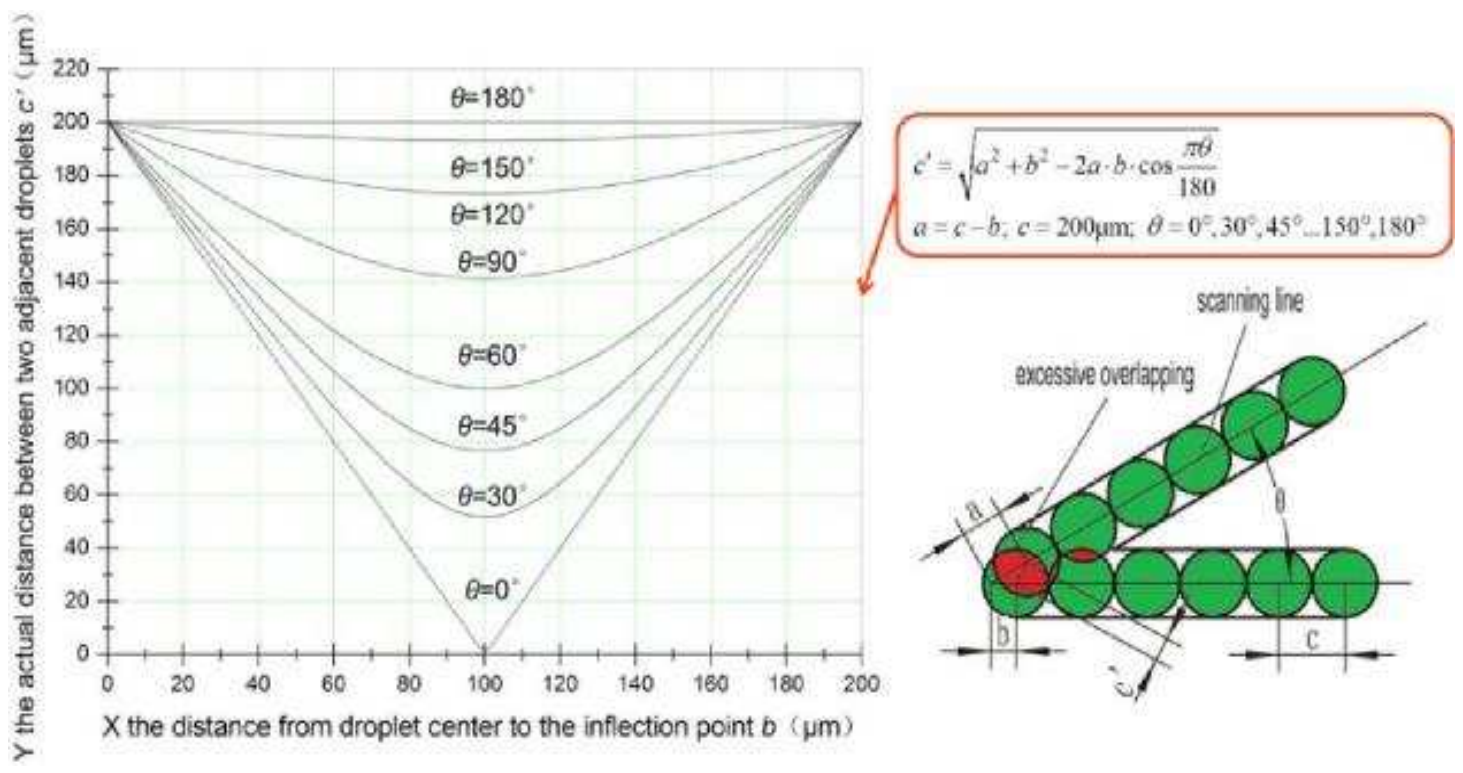

Fig. 2 The actual step distance under corner angle $\theta$ and the distance from the end droplet to inflection point $b$. 


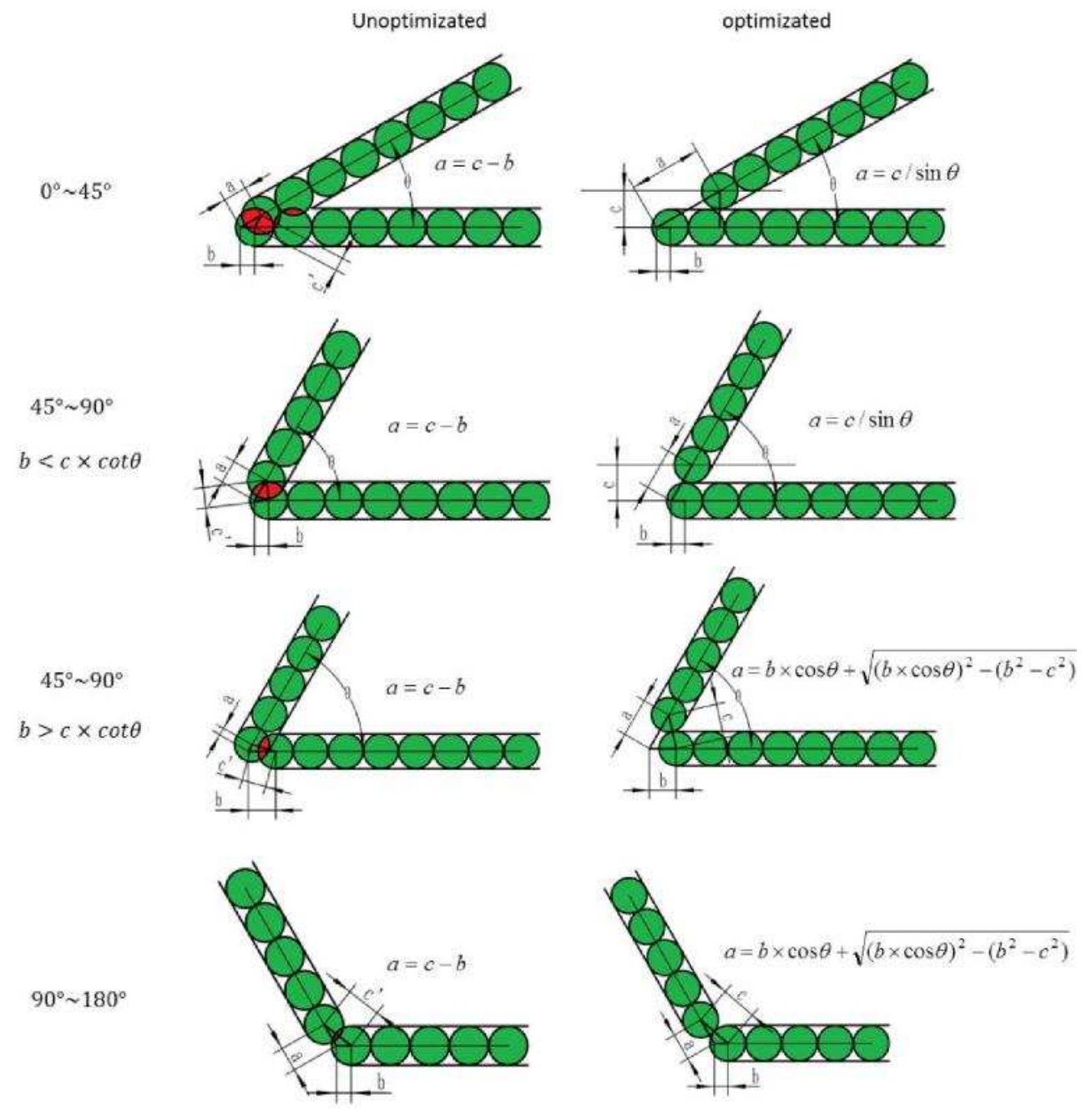

Fig. 3 Geometry control at different angles. 


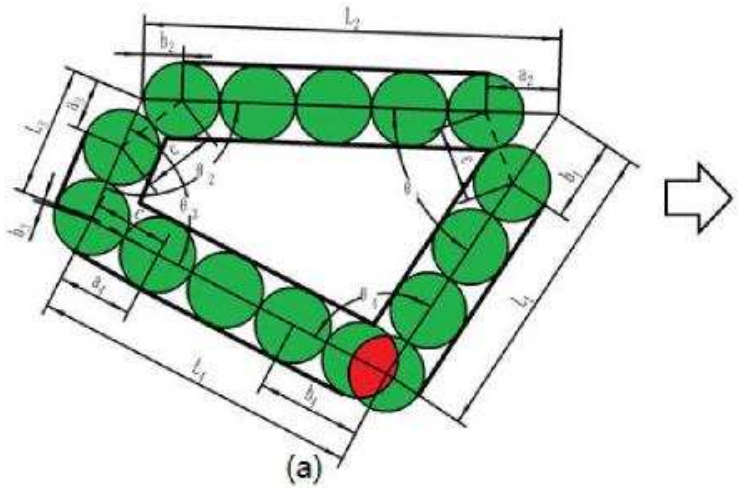

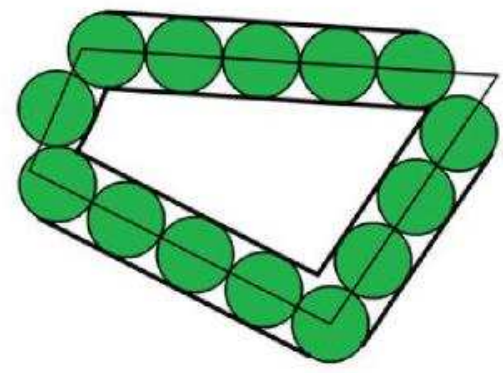

(b)

Fig. 4 Rearrange for droplets position of contour closure

(a)Excessive overlapping in the contour closure; (b) Contour formed by compensated step distance. 


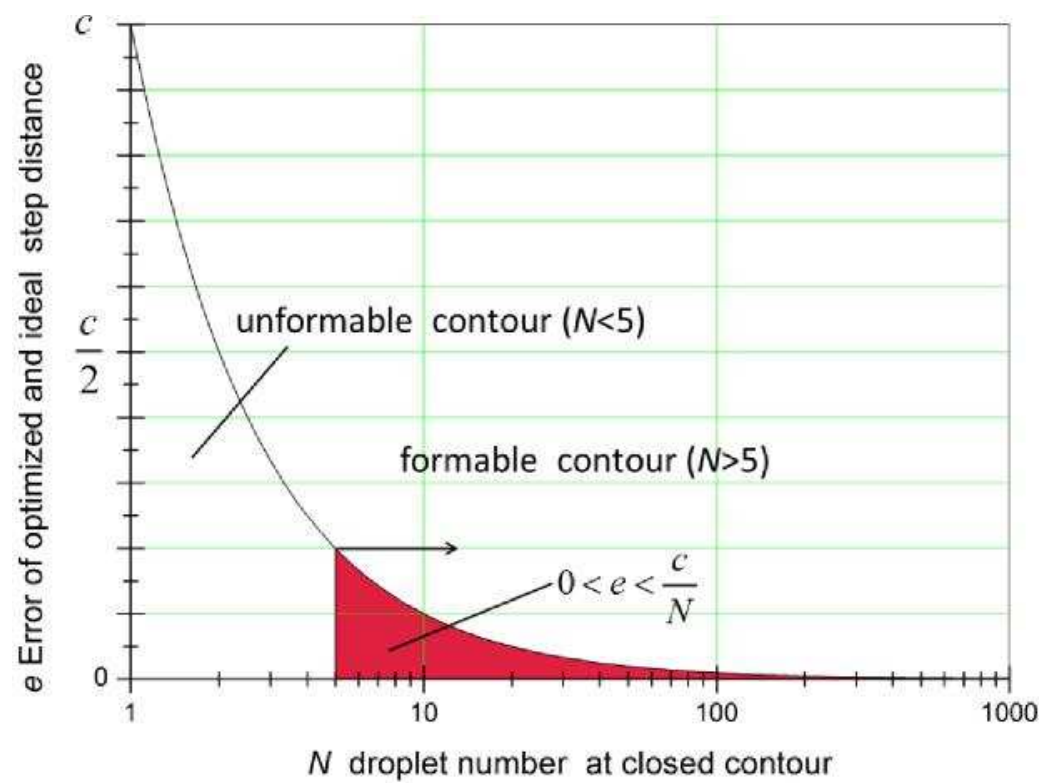

Fig. 5 Error of step distance after optimized. 


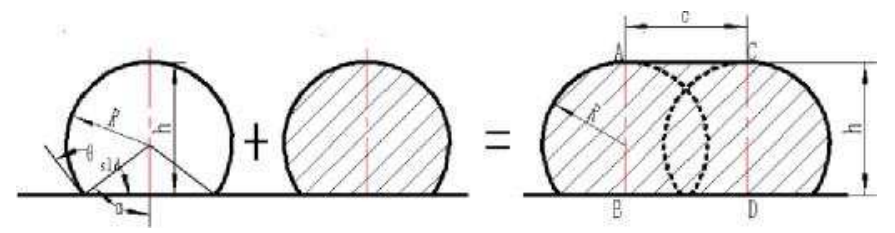

Fig. 6 Merge of droplets. 

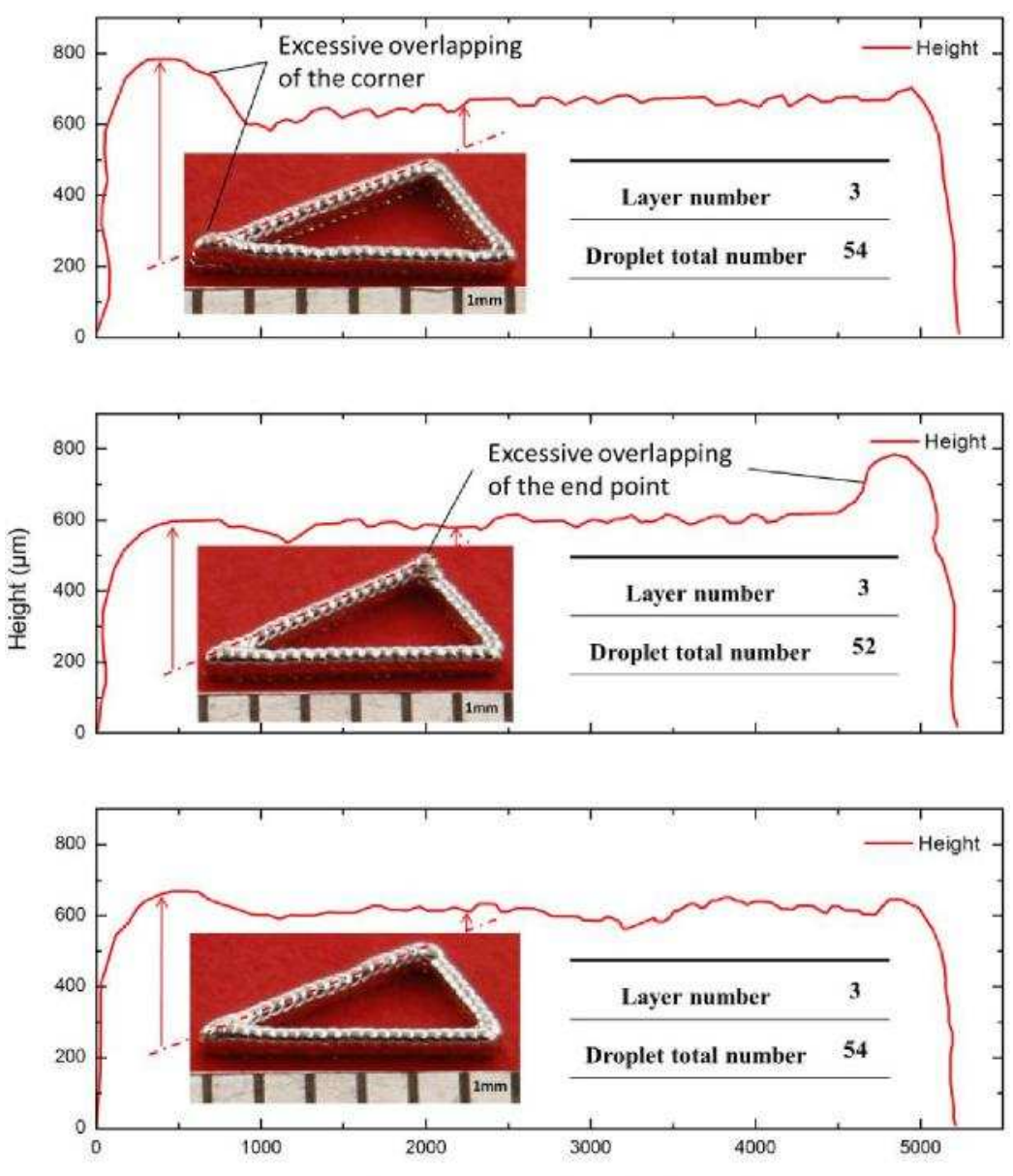

Fig. 7 optimization of a triangle sample.

(a) No optimization; (b) Corner optimization; (c) Contour optimization. 
Table 1 The related parameters of experiment environment

\begin{tabular}{ll}
\hline Parameter & Value \\
\hline Droplet material & Sn63\% Pb37\% \\
Droplet initial temperature $(\mathrm{K})$ & 573 \\
Substrate temperature $(\mathrm{K})$ & $293 \sim 298$ \\
Spraying back pressure $(\mathrm{kPa})$ & $3.0 \sim 5.0$ \\
Pulse width $(\mathrm{ms})$ & 1 \\
Deposition distance $(\mathrm{mm})$ & $1 \sim 5$ \\
Oxygen content of environment(PPM) & $50 \sim 60$ \\
Nozzle diameter $(\mu \mathrm{m})$ & 300 \\
\hline
\end{tabular}


Table 2 The related parameters of optimization calculation

\begin{tabular}{lllllll}
\hline \multirow{2}{*}{ Optimized method } & $\begin{array}{l}c / c c m p \\
(\mu \mathrm{m})\end{array}$ & $\begin{array}{l}e \\
(\mu \mathrm{m})\end{array}$ & $i$ & $m_{i}$ & $\begin{array}{l}b_{i} \\
(\mathrm{~mm})\end{array}$ & $\begin{array}{l}a_{i} \\
(\mathrm{~mm})\end{array}$ \\
\hline \multirow{3}{*}{ No optimization } & & & 1 & 20 & 0.146 & 0 \\
& 252.5 & 0 & 2 & 23 & 0.086 & 0.106 \\
& & & 3 & 11 & 0.056 & 0.166 \\
Corner optimization & 252.5 & 0 & 2 & 21 & 0.192 & 0.505 \\
& & & 3 & 10 & 0.183 & 0.292 \\
& & & 1 & 21 & 0.060 & 0 \\
Contour optimization & 245 & 7.5 & 2 & 22 & 0.129 & 0.489 \\
& & & 3 & 11 & 0.027 & 0.282 \\
\hline
\end{tabular}

